\title{
Design and application of electric power communication network comprehensive simulation training system
}

\author{
Lu Liu ${ }^{1, *}$, Xue $\mathrm{Hao}^{2}$, Lizhuo $\mathrm{Geng}^{2}$, Qi Gao ${ }^{2}$, Zhe $\mathrm{Liu}^{2}$, and Feng Gao ${ }^{1}$ \\ ${ }^{1}$ Beijing Kedong Power System Control Co.,Ltd, Beijing 100192, China \\ ${ }^{2}$ Hebei Electric Power Company Training Center, Shijiazhuang 050031, China
}

\begin{abstract}
To solve the problem that current communication network training tools have limited functions and can't effectively support the electric power communication network training, a new communication network comprehensive training simulation system is designed and implemented. This paper introduces the architecture of the system and function of each part. Then the simulation model principle is explained. The paper introduces the key technologies of 3D modeling, mechanism modeling, communication network scenario automatic generation and network fault simulation. Finally, the system application is introduced and the results show that the system builds a multi-functional training tool to meet the requirements of training and assessment for electric power communication network personnels.
\end{abstract}

\section{Foreword}

With the rapid development of Energy Inteconnection, the characteristics of power grid digitization, informatization and intellectualization have become increasingly prominent, and the role of electric power communication network, which provides reliable transmission channel for power grid operation, management, administration, marketing and other massive data, is becoming increasingly important ${ }^{[1-2]}$. New communication technology, equipment and system are constantly put into application in the power grid, which puts forward higher requirements for the ability of communication network O\&M (Operation and Maintenance) personnels. At present, the training centers of State Grid Corporation mainly rely on a small number of real equipments to build training network for communication training. There are not only problems of high construction and operation cost, but also other problems, such as small networking scale, no support for multi-person parallel operation, few fault analysis and treatment cases ${ }^{[3-4]}$. The existing communication simulation systems mainly simulates the receiving, processing and sending of data frames/packets between equipments. They don't have the hardware operation ability of communication equipments and cables, does not support the use of test instruments, and can not present the hardware status change under abnormal conditions, and the training effect is poor ${ }^{[5-7]}$. Therefore, it is of great significance to research and develop a

* Corresponding author: $\underline{\text { liulukd } @, 163 . c o m}$ 
comprehensive all-digital simulation training system which can simulate both threedimensional (3D) appearance and logic mechanism of various communication equipments, and fully reproduce the hardware status and network management information of equipment in normal/abnormal situation, so as to provide an efficient and flexible training tool of power communication.

In this paper, based on computer simulation, 3ds Max, Unity3 $\mathrm{D}^{[8-9]}$ and other technologies, a hierarchical system architecture of electric power communication network comprehensive simulation training system is designed, and an equipment simulation model is proposed to simulate both the hardware and functional characteristics of equipment. At the same time, the key technologies and implementation methods of 3D modeling, mechanism modeling, dynamic generation of network scenarios, network fault simulation are studied. The simulation training system has rich functions, its operation mode and result are consistent with the actual system. After application, it can fully meet the needs of communication network training, drilling, assessment and deduction for O\&M personnels.

\section{System architecture}

All-digital electric power communication comprehensive simulation training system adopts hierarchical architecture, which is divided into four layers from bottom to top: platform layer, model layer, function layer and application layer. Each layer is relatively independent to facilitate the expansion and modification of system functions.

The platform layer includes simulation support environment, 3D development engine, database and management system. The simulation support environment is developed based on high level architecture (HLA), which provides a transparent and efficient virtual support environment for each simulation module, and provides common services for the interaction between modules. The 3D development engine is based on Unity3D development engine, and combined with the requirements of communication equipment $3 \mathrm{D}$ modeling and $3 \mathrm{D}$ interaction. The database and management system includes real-time database and relational database, and provides a unified access interface to the database.

The model layer establishes the models corresponding to each equipment in the electric power communication network, including the models of transmission equipment, access equipment, data communication equipment, communication room, communication cable, distribution frame, test instrument and communication power supply. Each model has the same 3D appearance as the simulated equipment, supports the $3 \mathrm{D}$ operation, and realizes the communication logic function of the simulated equipment.

The function layer includes three functions: network management system simulation, fault simulation and automatic generation of network scenarios. The network management system simulation function simulates the software function of transmission network management system, access network and data communication network configuration tool, supports the same operation mode and has the same interaction result. The network scenario automatic generation function analyzes the script file, calls the relevant model, dynamically generates the three-dimensional network scenario, and starts the communication logic function to realize data processing and interaction. The fault simulation function establishes multi-type software and hardware fault models, and analyzes the fault trigger data, generate the fault phenomenon. The fault simulation function can also judge whether the trainees' action to deal with the fault is correct.

The application layer includes instructor system and trainee system. Instructor system generates and distributes the simulation configuration files, and monitors and manages the trainee system. Trainee system generates simulation network scenarios, starts data interaction between simulated equipments, and triggers faults. At the same time, the trainee 
system executes the control commands from the instructor system, and collects the operation records and uploads them to the instructor system.

\section{Key technologies and implementation methods}

\subsection{Communication equipment modeling}

In this system, the model structure and construction method of different equipment, cable, instrument, facilities are similar. Due to the complexity of hardware structure and logic function of SDH equipment, this paper takes SDH equipment as an example to introduce the structure and modeling method of communication equipment simulation model.

SDH equipment simulation model is a comprehensive simulation of the hardware and functional characteristics of real equipment. It not only realizes the 3D status display and $3 \mathrm{D}$ operation functions of $\mathrm{SDH}$ equipment, but also realizes the communication signal sending, receiving and processing functions. The structure of SDH equipment simulation model is shown in Figure 1. The real SDH equipment in the physical space is abstracted into the three-dimensional model and machanism model in the simulation space. Through the interaction of status attributes and status parameters, the two models form a unified SDH equipment simulation model.

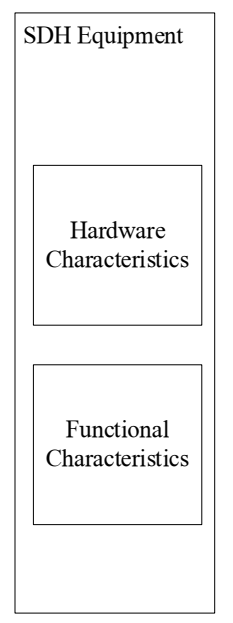

Physical Space

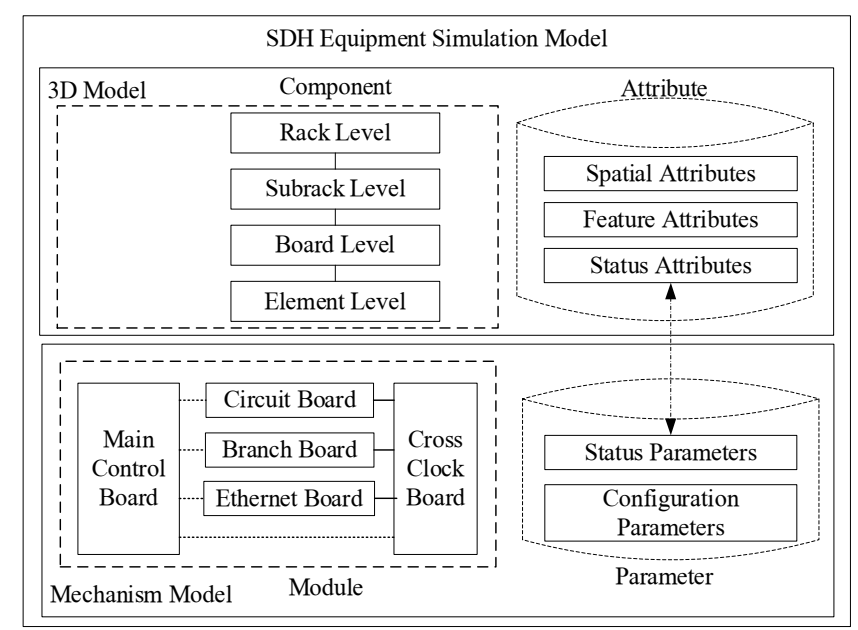

Simulation Space

Fig. 1. SDH equipment simulation model.

Different manufacturers and different types of SDH equipment have different hardware characteristics, but they can be abstracted as a 3D model composed of several 3D components. According to the physical inclusion relationship, 3D components are divided into four levels: Rack Level, Subrack Level, Board Level and Element Level (including port, switch and indicator). Each component has its own attributes, including spatial attributes (location, size, posture, etc.), feature attributes (type, supported operations, etc.), and status attributes (board-in-place status, indicator color, etc.).

The functional characteristics of SDH equipment can be abstracted as a mechanism model composed of five processing modules. Each processing module corresponds to a board type in $3 \mathrm{D}$ model to realize the data processing logic of the board. According to the configuration parameters, the communication data is exchanged between the modules, and the equipment status parameters are updated. 
In real SDH equipment, functional characteristics and physical characteristics are two closely linked parts. The change in one characteristic will cause change in another characteristic in real time. In this system, the status attributes and status parameters are stored in the real-time database, and the related data are associated, such as the SRV_Color attribute of Circuit Board 3D model_and Fault_Status parameter of Circuit Board mechanism model are associated. Through the synchronous updating of status attributes and status parameters, the $3 \mathrm{D}$ model and the mechanism model realize real-time collaborative simulation. For example, during the running of Circuit Board mechanism model, if abnormal situation is detected, then alarm is generated, and status parameter is changed (Fault_Staus=YES). The 3D model finds the change of status parameters, updates the associated status attribute (SRV_Color=RED), and displays the new status in 3D scenario (SRV light of Circuit Board turns red).

\subsubsection{D modeling}

The process of establishing 3D model of SDH equipment is: 1) Measuring physical properties and collecting operation data of equipment. 2) Various 3D components with physical appearance characteristics are created by $3 \mathrm{ds}$ Max modeling tool at four levels: Rack, Subrack, Board and Element, and operation animation is created at the same time. 3) In Unity3D, components are assembled as 3D models of equipment according to assembly rules, and effect rendering and attribute settings are made. 4) The corresponding operating procedures of 3D components are developed by using $\mathrm{C} \#$ language. 5) The $3 \mathrm{D}$ model is debugged, optimized and released, and stored in the model database.

\subsubsection{Mechanism modeling}

In order to flexibly and accurately reproduce the data processing logic, alarm generation and propagation logic of SDH equipment under various normal and abnormal situations, the system uses the data processing and interaction algorithm based on ITU-T specification to establish the logic mechanism model. According to SDH specification, the main internal functional components of the equipment are divided into 18 basic logic functional units. Each functional unit independently realizes some processing process. In this system, according to the logical function of SDH equipment from main manufacturers (Huawei, ZTE, etc.), the logical functional units are combined into five modules: Circuit Board, Branch Board, Ethernet board, Cross Clock Board and Main Control board. The five modules correspond to the five kinds of boards in 3D model. Through the data interaction between modules, the five modules constitute the logic model of SDH equipment, and realize the signal receiving and sending, data multiplexing and mapping, overhead analysis, alarm generating, and cross connection functions of the SDH equipment.

\subsection{Automatic generation of communication network simulation scenarios}

The communication network simulation scenario is composed of models of communication room, communication equipment, distribution frame, cable, test instrument, etc. In different training cases, network topology, number of equipments, equipment type are different, and the corresponding network simulation scenario is different. If the communication network simulation scenario is built manually, the efficiency is low. In this system, an automatic generation method of communication network simulation scenario is proposed, which reads and interprets the network simulation script file, loads various models automatically, and 
generates communication network scenario. By modifying the script file, various communication network simulation scenarios can be generated flexibly and dynamically.

Communication network simulation script file is a self-defined XML format file, including three types of information: network scenario data, configuration data, fault trigger data. The network scenario data defines the communication room, rack, equipment and connection information related to the scenario. Its structure is divided into four layers. The first layer defines the room name, room type and number of racks. The second layer defines the rack name, rack type, rack coordinates, number of equipments, and number of test instruments. For different types of equipment, the content of the third layer definition is different: for example, for communication equipment, the third layer defines the equipment type, equipment location and board configuration; for optical distribution frame (ODF) and digital distribution frame (DDF), the third layer defines the frame type, frame location and number of ports; for test instrument, the third layer defines the instrument type. The fourth layer defines the cable type, cable identification and connection location.

The automatic scenario generation function uses the top-down sequence to scan the network scenario data in a hierarchical manner: load the corresponding communication room model according to the first layer data to generate the $3 \mathrm{D}$ communication room; load the corresponding 3D rack models according to the second layer data, and place the models in the $3 \mathrm{D}$ communication room according to the location coordinate information; load the corresponding $3 \mathrm{D}$ equipment models according to the third layer data and place the models in the corresponding rack, then load the corresponding test instrument models and placed them in the toolbox; according to the fourth layer data, the corresponding optical fiber, $2 \mathrm{M}$ cable, Ethernet cable, communication power cable model, etc. are loaded, and the corresponding ports of equipment or distribution frame are connected according to the connection information. After the model is loaded successfully, the configuration data in the communication network simulation script file is read, and the respective logical models are started to receive, send and process the simulation communication data.

\subsection{Communication network fault simulation}

Based on the dynamically generated network simulation scenario, the fault simulation function and equipment model are coordinated to realize the communication network fault simulation. The fault simulation function first reads and interprets the fault triggering data in communication network simulation script file. The fault trigger data includes information such as the number of faults, the type of each fault and the fault point. According to the type of each fault, corresponding fault model is found to obtain the processing logic change data and parameter change data caused by the fault. Then according to the fault point information, the specific equipment and its mechanism module where the fault is located is found, and the module is informed to run the changed logic program based on the new parameters. After processing the overhead field of communication data among the modules, related alarm is generated automatically and sent to the network management simulation function, and the status parameters of mechanism model are changed.

The 3D model and network management simulation program are combined to realize the presentation of fault phenomenon. The $3 \mathrm{D}$ model reads the changes of status parameters in the mechanism model, and presents the hardware fault status in the $3 \mathrm{D}$ scenario, such as the board indicator light's color change and the buzzer on. The network management simulation function monitors the generation of alarm data, and displays the corresponding alarm information in the "browse current alarm" window, such as alarm level, alarm name, alarm source, location information, alarm occurrence time, clearing status, etc. 


\section{System deployment and application}

The electric power communication network comprehensive simulation training system has been successfully deployed and applied in the several provincial training centers of State Grid, such as Hebei, Shanxi and Jiangxi training center. Through the simulation training system, the trainees drill the training cases related to equipment operation, hardware maintenance, data configuration and fault handling. The training case truly reproduces the different kinds of communication network scenarios. It supports the simultaneous simulation of 2-100 equipments, supports various network topologies, and supports 18 kinds of communication network fault, such as communication link interruption, optical fiber degradation, fan failure, Circuit Board fault, Branch Board fault, Cross board fault, protection fault and data configuration error, etc.

\section{Conclusion}

In this paper, the unique $3 \mathrm{D} /$ mechanism integrated communication equipment modeling technology, communication network simulation scenario automatic generation technology and communication network fault simulation technology are used to design and develop an electric power communication network comprehensive simulation training system, which has been applied in several provincial training centers of State Grid Corporation of China. The system solves the problem that the existing communication simulation system can not simulate the three-dimensional scenario and equipment logic mechanism at the same time, and can not present the hardware status and network management information at the same time. The system solves the problem of high cost of building a real training network, and it supports flexible triggering of multiple types of communication network faults. Through the use of the system, trainees can quickly learn and master the communication equipment O\&M skills and configuration skills, improve the fault analysis and processing ability, shorten the training cycle and reduce the training cost. At the same time, the architecture and technical implementation method of the system also provide reference for the design and development of simulation training system in other fields.

\section{References}

1. J.Y. Wang, T.J. Pu, J. Tong, Power Syst. Technol. 18, 1 (2020)

2. J. Hao, X. Chen, J. Tong, Elec. Technol. \& Soft. Engi. 1, 7 (2020)

3. J.F. Ge, Electric Power Communication (China Electric. Power Press, Beijing, 2010)

4. Y.J. Li, Tele. Commun. Technol. 3, 74 (2012)

5. Z.W. Liu, J. Huang, S.B. Tong, Elec. Power Infor. Commun. Technol. 13, 70 (2015)

6. J.H. Yan, B.W. Chen, Optical Technol. 3, 11 (2005)

7. T.F. Zhang, B. Bi, J. Wang, J.H. Lv, Comput. CD Soft. Appli. 11, 252 (2013)

8. G.D. Ren, L.H. Chen, X.F. Tao, X.X. Fang, Comput Syst Appl. 9, 85 (2013)

9. C.S. Pan, J.Y. Yang, Mining Safety Enviro. Prot. 4, 32 (2014) 